$$
\begin{aligned}
& \text { اختبار اثر عدم الاستقرار على نمط انفاق المستهلك العراقي } \\
& \text { م. رباب عبد الرضا } \\
& \text { أ. د. أموري هادي كاظم } \\
& \text { البكري } \\
& \text { جامعة بغداد/ كلية الادارة والاقتصاد } \\
& \text { قسم الاحصاء }
\end{aligned}
$$

1 - الاقدهية

يحتل موضوع الاستهلاك اهمية كبيرة في الدراسات الاقتصادية في حالتي السلم والحرب وذلكاتك لارتباط

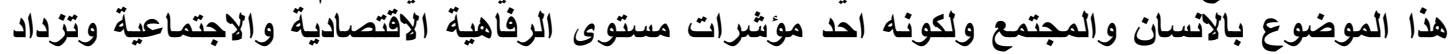

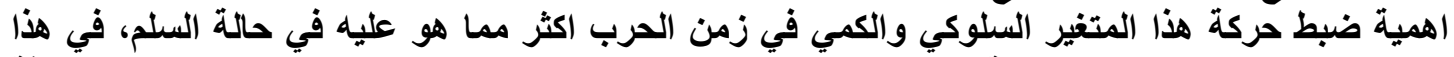

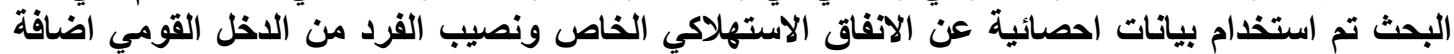

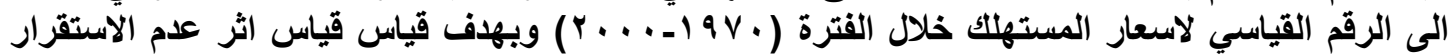

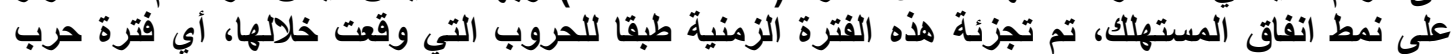

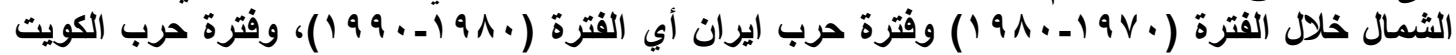

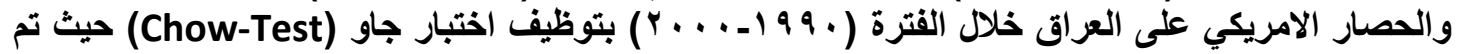

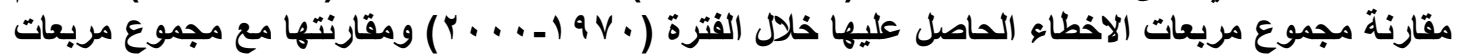

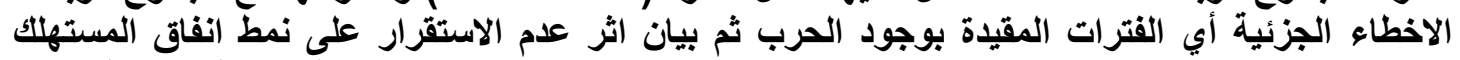

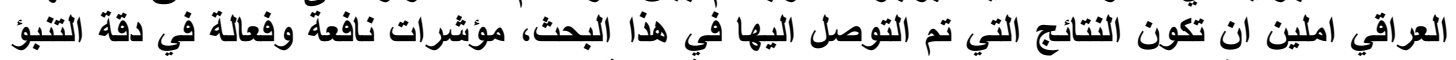

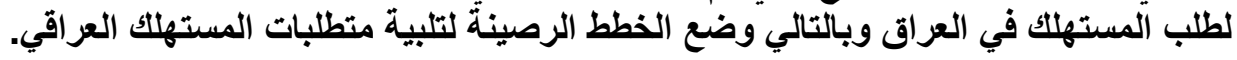

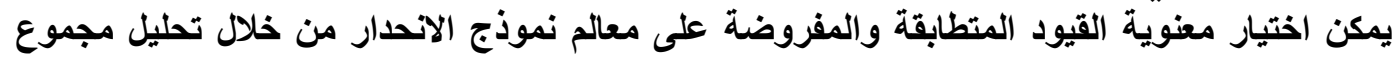

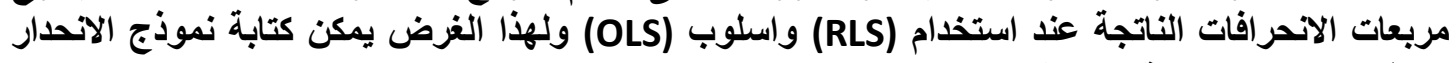

$$
\begin{aligned}
& y_{1}=x_{1} B_{1}+\mu_{1} \\
& y_{2}=x_{2} B_{2}+\mu_{2}
\end{aligned}
$$

$$
\begin{aligned}
& \text { حيث: }
\end{aligned}
$$

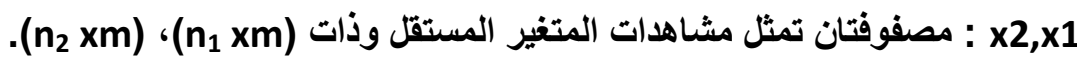

$$
\begin{aligned}
& \text { (m x1). } \\
& \text { عإن } \\
& \text { علما بان: } \\
& \mu_{1} \sim N\left(0, \sigma^{2} I\right) \\
& \mu_{2} \sim N\left(0, \sigma^{2} I\right) \\
& \operatorname{COV}\left(\mu_{1}, \mu_{2}\right)=0 \\
& y=x B+\mu \\
& y=\left[\begin{array}{l}
y_{1} \\
y_{2}
\end{array}\right], x=\left[\begin{array}{cc}
x_{1} & 0 \\
0 & x_{2}
\end{array}\right], B=\left[\begin{array}{l}
B_{1} \\
B_{2}
\end{array}\right], \mu=\left[\begin{array}{l}
\mu_{1} \\
\mu_{2}
\end{array}\right]
\end{aligned}
$$$$
\text { و } n=n_{1}+n_{2}
$$ 
ويتلخص اسلوب اختبار مدى تاثير القيود في النموذجين المذكورين اعلاه من خلال تحليل القيود

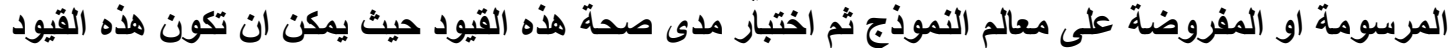

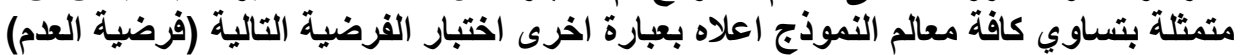
Ho:

$$
B_{01}=\dot{B}_{02}=\cdots=B_{0 p}
$$

$$
B_{t 1}=B_{t 2}=\cdots=B_{t p}
$$

t=2,3,...,m-1 حيث ان p تمثل عدد القيود و

$$
b_{L S}=\left(x^{\prime} x\right)^{-1} x^{\prime} y
$$

من النموذج اعلاه يمكن ان نجد مباشرة تقدير للمعالم بالثكل التالي:

$$
S=e^{\prime} e=\left(y-x_{b L S}\right)^{\prime}\left(y-x_{b L S}\right)
$$

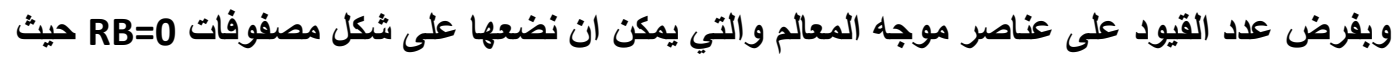
R تمثل من مرتبة او مصفوفة بالثكل التالي

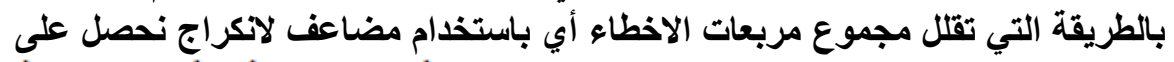

$$
\begin{aligned}
& \mu^{\prime} \mu=(y-x B)^{\prime}(y-x B)-2 \lambda R B \\
& \frac{\partial \mu^{\prime} \mu}{\partial B}=-2 x^{\prime} y+2 x^{\prime} x b_{R L S}-2 \lambda^{*} R=0 \\
& \frac{\partial \mu^{\prime} \mu}{\partial \lambda}=-2 R b_{R L S}=0 \\
& b_{R L S}=b_{L S}+\left(x^{\prime} x\right)^{-1} R^{\prime}\left[R\left(x^{\prime} x\right)^{-1} R^{\prime}\right]^{-1} R b_{L S} \\
& \text { اما مجموع مربعات الانحرافات الناتج من طريقة (RLS) يمكن الوصول اليها بالثكل التالي: } \\
& S^{*}=e^{* \prime} e^{*}=\left(Y-x b_{R L S}\right)^{\prime}\left(Y-x b_{R L S}\right) \\
& =y^{\prime} y-b_{R L S}^{\prime} x^{\prime} y-y^{\prime} x b_{R L S}+b_{R L S}^{\prime} x^{\prime} x b_{R L S} \\
& \text { وبالتعويض عن صيغة (bLS) } \\
& S^{*}=y^{\prime} y-y^{\prime} x b_{R L S}-b_{R L S} R^{\prime}\left[R\left(x^{\prime} x\right)^{-1} R^{\prime}\right]^{-1} R b_{L S} \\
& E\left\{b_{R L S}^{\prime} R^{\prime}\left[R\left(x^{\prime} x\right)^{-1} R^{\prime}\right]^{-1} R b_{L S}\right\}=0 \\
& \therefore S^{*}=y^{\prime} y-y^{\prime} x b_{R L S} \\
& \text { وبالتعويض مرة اخرى عن قيمة (bLS } \\
& S^{*}=y^{\prime} y-y^{\prime} x b_{L S}-b_{L S} R^{\prime}\left[R\left(x^{\prime} x\right)^{-1} R^{\prime}\right]^{-1} R b_{L S} \\
& S^{*}=S+b_{L S}^{\prime} R^{\prime}\left[R\left(x^{\prime} x\right)^{-1} R^{\prime}\right] R b_{L S} \\
& S^{*}-S=b_{L S}^{\prime} R^{\prime}\left[R\left(x^{\prime} x\right)^{-1} R^{\prime}\right] R b_{L S} \\
& \text { وتحت فرضية العام (RB=0) يمكن ايجاد توزيع الفرق بين مجموع مربعات البواقي هذه وكالاتي: } \\
& R b_{L S}=R\left(x^{\prime} x\right)^{-1} x^{\prime} y \\
& R b_{L S}=R\left(x^{\prime} x\right)^{-1} x^{\prime}(x B+\mu) \\
& R b_{L S}=R\left(x^{\prime} x\right)^{-1} x^{\prime} \mu
\end{aligned}
$$




$$
\begin{aligned}
& S^{*}-S=\mu^{\prime} x\left(x^{\prime} x\right)^{-1} R^{\prime}\left[R\left(x^{\prime} x\right)^{-1} R^{\prime}\right]^{-1} R\left(x^{\prime} x\right)^{-1} x^{\prime} \mu \\
& S^{*}-S=\mu^{\prime} A_{1} \mu \\
& A_{1}=x\left(x^{\prime} x\right)^{-1} R^{\prime}\left[R\left(x^{\prime} x\right)^{-1} R^{\prime}\right]^{-1} R\left(x^{\prime} x\right)^{-1} x^{\prime}
\end{aligned}
$$

حيث Idempotent مصفو IA منى انها مصفوفة مربعة ومن الرتبة من هذه الخاصية يتضح

$$
\begin{aligned}
& S^{*}-S=b^{\prime}{ }_{L S} R^{\prime}\left\lfloor R\left(x^{\prime} x\right)^{-1} R\right] R b_{L S^{\sim}} \sim \sigma^{2} X^{2}{ }_{(K)} \\
& S=(y-x b)^{\prime}(y-x b) \\
& S=\mu^{\prime} A_{2} \mu \\
& A_{2}=\left[I-x\left(x^{\prime} x\right)^{-1} x^{\prime}\right] \\
& S=y^{\prime} y-y^{\prime} x b_{L S^{\sim}} \sim \sigma^{2} X^{2}{ }_{(n-2 m)} \\
& \therefore f(k, n-2 m)=\frac{\sigma^{2} X^{2}{ }_{K}}{\sigma^{2} X^{2}{ }_{(n-2 m)}}=\frac{S^{*}-S / P}{S /(n-2 m)}
\end{aligned}
$$

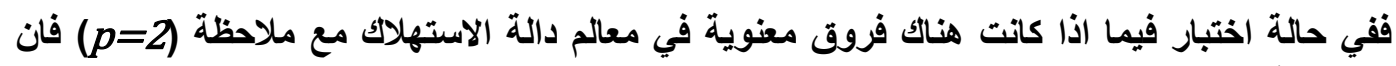

$$
\begin{aligned}
& f_{m+1, n-p(m+1)}=\frac{S^{*}-S / m+1}{S / n-p(m+1)} \\
& \text { اما اذا كانت p>2 او p=3 فان صيغة الاختبار تكون كما يلي } \\
& f_{(m+1),(p-1), n-p(m+1)}=\frac{S^{*}-S /(m+1)(p-1)}{S /(n-p)(m+1)}
\end{aligned}
$$

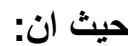

p مثثل عدد القيود المستقلة والمستخدمة في الفرضية المطلوب اختبارها

(m+1)

S: Sجموع مربعات الاخطاء الحاصل عليها من الانحدار الخطي الغير مقيد URLS او الانحار الخطي

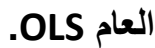

S مجموع مربعات الاخطاء الحاصل عليها الانحدار الخطي المقيد.

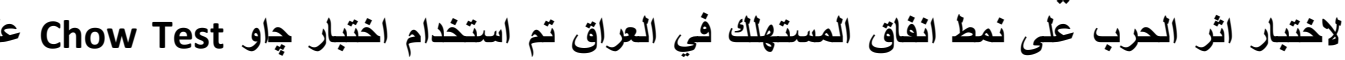

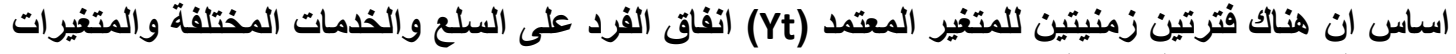

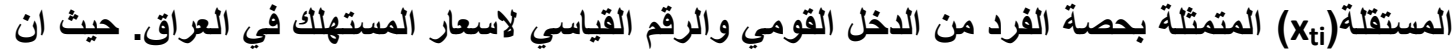

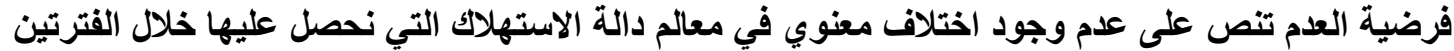

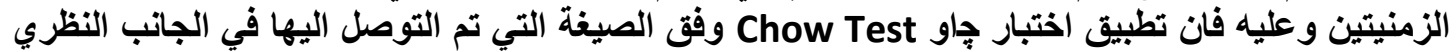

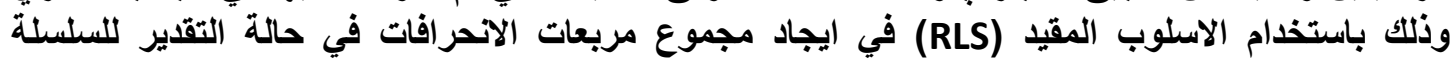
الزمنية المقيدة ومقارنتها مع السلسلة الزمنية غيلة غير المقيدة فيوع 


$$
\begin{aligned}
& H_{0}=B_{1}=B_{2}=0 \\
& H_{1}=B_{1} \neq B_{2} \neq 0
\end{aligned}
$$

1- $1970-2000$

$\hat{y}_{t}=322.912-0.00244 \hat{x}_{t}+1.959 \hat{Z}_{t}$

S.E(588.72256), $R^{2}(1.000), f(163925.8), \sigma^{\wedge 2}$ (346594.247)

D. $w(1.623), \eta i(0.0027), \mu p c(0.00244)$

رفض فرضية العدم والقبول بالفرضية البديلة الفروق معنوية في معالم دالة الاستهلاك للفترة اعلاه.

\section{2- 1970-1980}

$\hat{y}_{t}=-281.737-0.0167 \hat{x}_{t}+18.031 \hat{Z}_{t}$

S. $E(18.33768), R^{2}(0.961), f(98.09), \sigma^{\wedge 2}(336.27)$

D. $w(1.185), \eta i(0.49), \mu p c(0.167)$

رفض فرضية العدم والقبول بالفرضية البديلة الفروق معنوية في معالم دالة الاستهلاك للفترة اعلاه.

3- 1980-1990

$\hat{y}_{t}=-131.564-0.00197 \hat{x}_{t}+9.361 \hat{Z}_{t}$

$S . E(80.19277), R^{2}(0.95), f(75.242), \sigma^{\wedge 2}(6430.88)$

D. $w(1.797), \eta i(0.003), \mu p c(0.00197)$

رفض فرضية العدم والقبول بالفرضية البديلة الفروق معنوية في معالم دالة الاستهلاك للفترة اعلاه.

4- $1990-2000$

$\hat{y}_{t}=691.496-0.00229 \hat{x}_{t}+1.951 \hat{Z}_{t}$

S. $E(1014.47), R^{2}(1.000), f(29573.7), \sigma^{\wedge 2}(1029161.8)$

D. $w(2.084), \eta i(0.0025), \mu p c(0.0022)$

رفض فرضية العدم والقبول بالفرضية البديلة الفروق معنوية في معالم دالة الاستهلاك للفترة اعلاه. 


$$
\begin{aligned}
H_{0} & =B_{01}=B_{02}=\cdots=B_{O P} \\
& =B_{t 1}=B_{t 2}=\cdots=B_{t p}
\end{aligned}
$$

$$
H_{0}: R B=0
$$

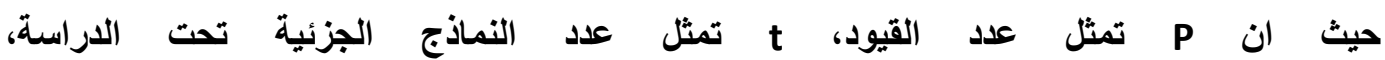

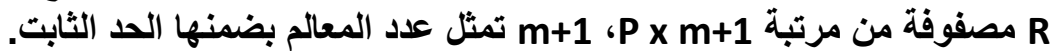

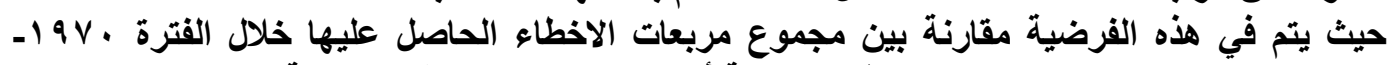

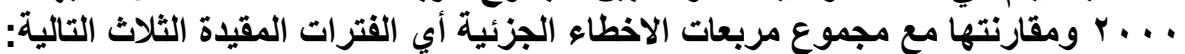

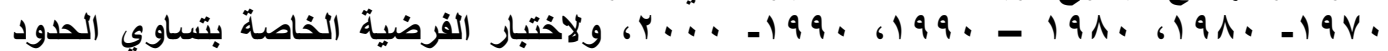

$$
H_{0}=B_{01}=B_{02}=B_{03}
$$
الثابتة في النماذج الجزئية الثلاث، أي ان:

\section{F Chow $=116492.7476$}

$$
\text { حيث ان P=3 , P=31=9 }
$$

وبتطبيق الصيغة الخاصة باختبار Chow نجد ان

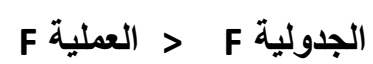

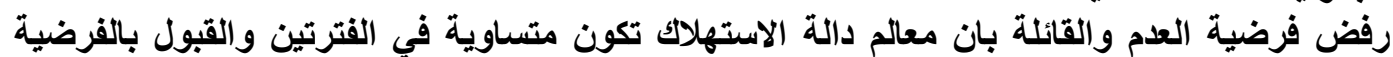

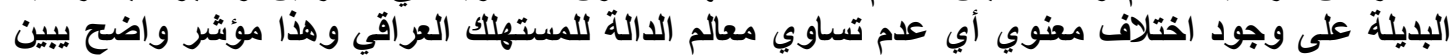

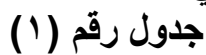
اثر عدم الاستقرار على نمط انفاق المستهلك العراقي.

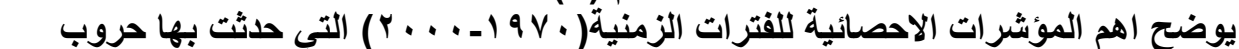

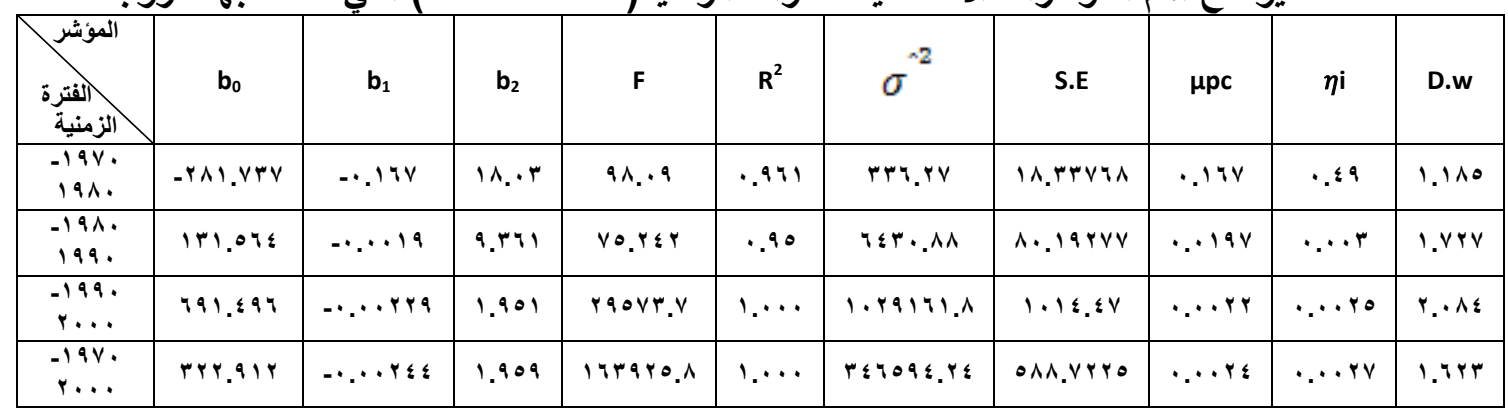


من المعلوم ان الاستهلاك الخاص يشكل احد المكونات الاساسية للاخل القومي في البلا، فعندما يطرا

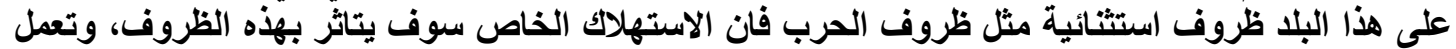

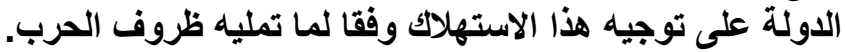

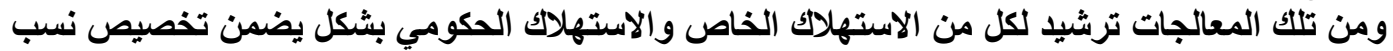

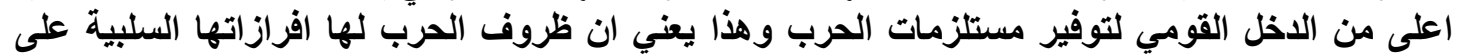

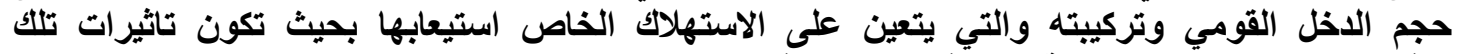

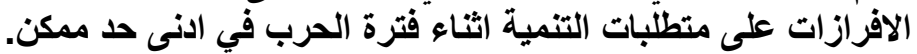

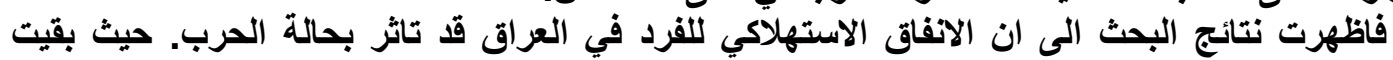

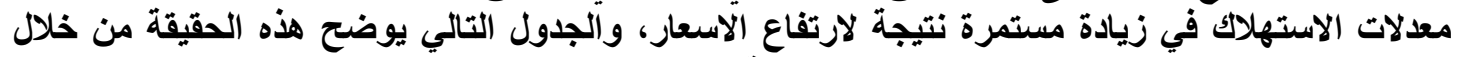

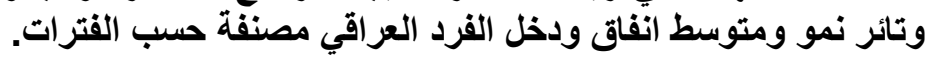

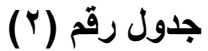

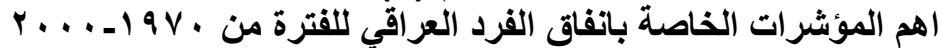

\begin{tabular}{|c|c|c|c|c|}
\hline المؤشر الفترة الزمنية & وتليرة النمو & $\begin{array}{c}\text { متوسط (نفاق الفرد } \\
\text { (دينار) }\end{array}$ & وتيرة النمو & 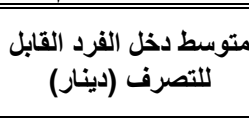 \\
\hline $19 V \cdot-1911$ &. .197 & 104 &. .471 & $\varepsilon r r$ \\
\hline $1911-1991$ &. .170 & VIV & $\because \varepsilon$ & N9r \\
\hline $1991-Y \ldots$ & .VYr & 90.04 & $\because \wedge \neg \wedge$ & $1 \cdot \vee \wedge \vee 7$ \\
\hline 19V.-r... & . RYT & r^q१ए & $\because$ YVO & rOYYV \\
\hline
\end{tabular}

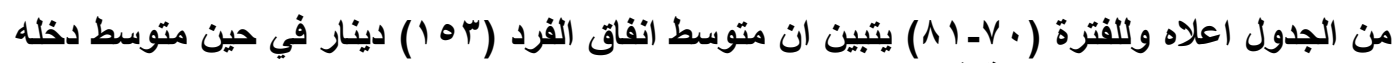

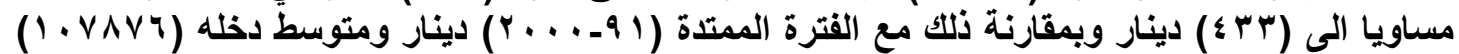

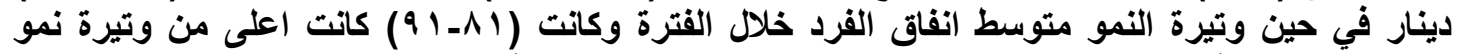

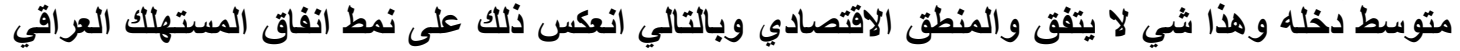

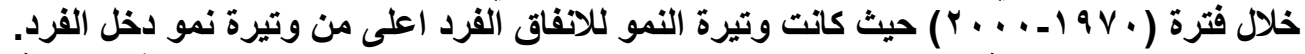

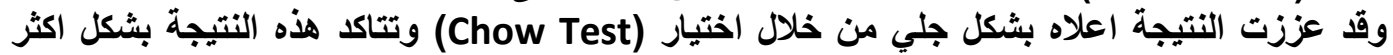

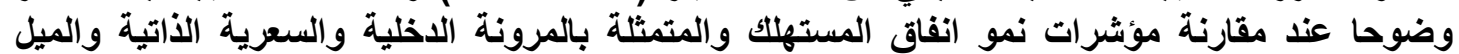

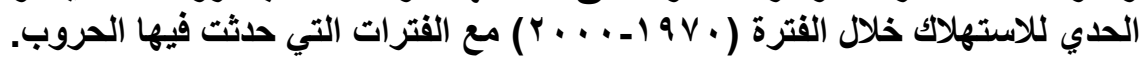


1- Chow, Gregory G., "Test of Equality between Sets of-Coefficient in tow Liner Regressions', Econometric vol. 28, July, 1990.

2- Mardia, K.V. and Kont, J. I and Bibby, J. N., " Multivariate and Analysis", Acadmic Press, Landon, 1971.

3- Theil, H., "On the Use of Incomplete Prior Informition in Regression Analysis", JASA, June, 1963.

ا- كاظم، د. اموري هادي، "استخدام النماذج المقيدة في تحليل دالة الاستهلاك"، مجلة الاقتصادي العدد

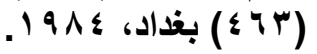

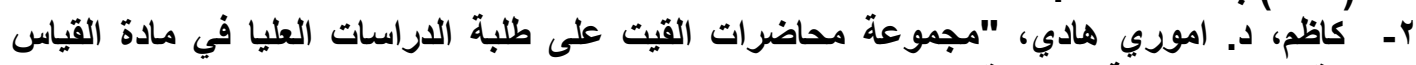

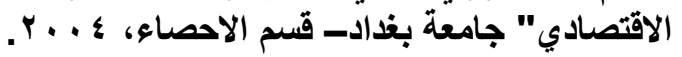

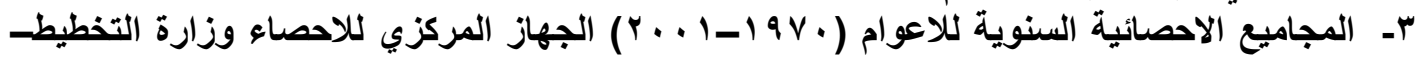
بغداد- الكراق. 